for patient and disease characteristics. According to DAS28 scores, disease activity, the cohort was divided into four groups: remission (Rem), low disease activity (LDA), moderate disease activity (MDA) and high disease activity (HDA).T2T, achieving a DAS28 score lower than 2.6 (Rem) or below 3.2 (LDA), is the main management strategy recommended by ACR and EULAR.

Results: From Jun 2014 to , Jan 2018 a total of 24,731 RA patients from 486 centres in China participated in the study. The mean age was $49.28 \pm 16.08$ (18 to 99) years and the median disease duration was 18.30 months. All patients performed selfassessment of DAS28, HAQ and morning stiffness time totally for 30358 times. Proportion of patients in Rem, LDA, MDA and HDA was $18 \%, 13 \%, 45 \%$ and $24 \%$ respectively at baseline. Of which, 3492 patients performed repeated assessment for 11251 times. Proportion of patients in Rem, LDA, MDA and HDA changed into $44 \%, 18 \%, 31 \%$ and $7 \%$ at the last assessment. The proportion of T2T at the last assessment was significantly higher than that of baseline significantly $(p<0.001)$. According to the assessments, the rate of T2T from baseline to 3 months, 6 months, 9 months, 12 months and over 12 months were $31 \%, 47 \%, 56 \%, 58 \%$, $61 \%$ and $62 \%$ (see figure 1). With the increase of the times of self-assessment, the T2T rate was significantly improved $(p<0.001)$.

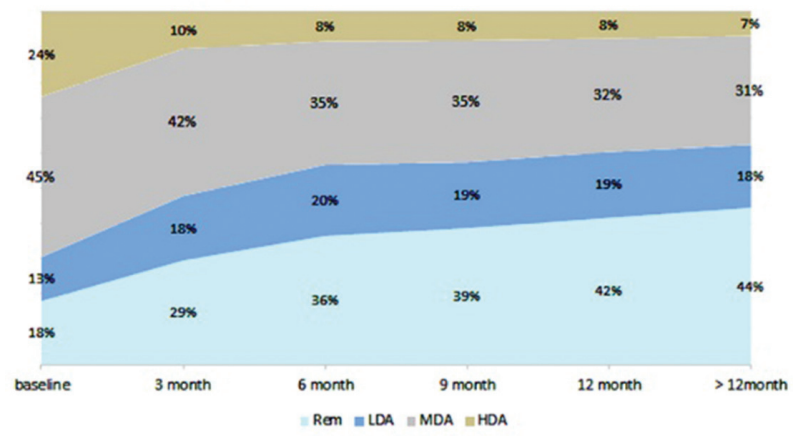

Abstract FRI0049 - Figure 1. The times of repeated self-assessment and the proportion of DAS28 stratification

Conclusions: Under repeated self-assessment of DAS28 using SSDM, RA patients can achieve better T2T result. Though empowering patients, SSDM can assist rheumatologist to rationally adjust treatment for RA patients.

Disclosure of Interest: None declared

DOI: 10.1136/annrheumdis-2018-eular.6412

\section{FRI0050 \\ TOCILIZUMAB ACHIEVES BETTER REPAIR OF FOCAL BONE EROSIONS THAN TUMOUR NECROSIS FACTOR INHIBITION IN RA PATIENTS - DATA FROM THE PROSPECTIVE REBONE STUDY ON EROSION REPAIR}

G. Schett ${ }^{1}$, S. Finzel ${ }^{2}$, S. Kraus ${ }^{1}$, A. Regensburger ${ }^{1}$, R. Kocijan ${ }^{3}$, J. Rech ${ }^{1}$. ${ }^{1} F A U$ Erlangen-Nuremberg, Erlangen; ${ }^{2}$ University of Freiburg, Freiburg, Germany; ${ }^{3}$ Medical University of Vienna, Vienna, Austria

Background: Focal bone erosions are considered as markers for irreversible structural damage in patients with rheumatoid arthritis (RA). Several studies have suggested that limited repair of focal bone erosion can occur but no study has so far compared the effect of different biological disease modifying anti-rheumatic drugs (bDMARDS) on erosion repair.

Objectives: To compare focal bone erosion repair in RA patients treated with interleukin-6 receptor inhibitor (tocilizumab, TOC) with patients receiving tumour necrosis factor inhibitors (TNFi)

Methods: Prospective non-randomised observational study in 66 erosive RA patients with active disease (DAS28-ESR>3.2) and inadequate response to methotrexate receiving treatment with TOC monotherapy $(n=33)$ or TNFi in combination with methotrexate $(n=33)$ for 52 weeks. Patients received high-resolution peripheral quantitative computed tomography (HR-pQCT) of the MCP and wrist joints of the dominant hand at baseline and after 52 weeks. Volume (in $\mathrm{mm} 3$ ) of the largest ("sentinel") erosion in the MCP joints and in the wrist was assessed by two readers blinded for treatments and for the sequence of the images. Demographic (sex, age, body mass index, smoking, Charlson comorbidity index) and disease specific parameters (disease duration, ACPA and RF status) were assessed at baseline and activity scores (DAS28, SDAI, CDAI, HAQ) at baseline and every three months thereafter.

Results: Groups were balanced for age, sex, BMI and comorbidities as well as disease duration, disease activity, functional state, autoantibody status and bone damage at baseline. TOC (DAS28-ESR: baseline: $6.2 \pm 0.5 ; 52 \mathrm{wk}: 2.3 \pm 1.0$ ) and TNFi (DAS28-ESR: baseline: $6.3 \pm 0.6$; $52 \mathrm{wk}$ : $2.8 \pm 1.2$ ) significantly reduced disease activity to a similar extent, achieving DAS28 remission in 22/33 and 19/33 patients, respectively, after 52 weeks. Volumes of the sentinel erosions significantly decreased in the MCP joints of TOC patients $(-1.0 \pm 1.1 \mathrm{~mm} 3)$, while remaining stable in TNFi treated patients $(-0.05 \pm 0.9 \mathrm{~mm} 3)$ (TOC vs. TNFi: $\mathrm{p}<0.001)$. Similar effects were observed in the wrist (TOC: $-2.9 \pm 5.6 \mathrm{~mm}^{3} ;-0.08$ $\pm 4.1 \mathrm{~mm}^{3}$ ) with significant differences between the two groups $(\mathrm{p}=0.0017)$. Erosion repair was particularly frequent in RA patients reaching fast remission within the first 3 months of treatment.

\section{Erosion repair in the metacarpophalangeal joints of tocilizumab treated RA patients after 52 weeks}
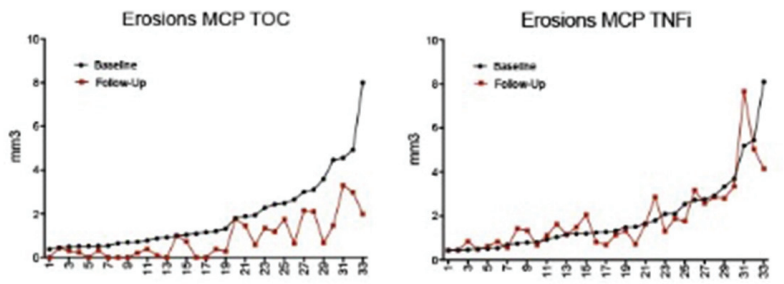

$Y$ axis shows the volume of the sentinel erosion at baseline (black circles) and 52-weeks follow-up (red squares), $x$-axis the patient numbers ( $N=33$ )

Abstract FRI0050 - Figure 1. Erosion repair in the metacarpophalangeal joints of tocilizumab treated RA patients after 52 weeks

$Y$ axis shows the volume of the sentinel erosion at baseline (black circles) and 52 weeks follow-up (red squares), $x$-axis the patient numbers $(n=33)$

Conclusions: The REBONE study shows that TOC has higher efficacy than TNFi to repair existing bone erosions in patients with RA. In contrast, the effects of TOC and TNFi on the inflammatory symptoms of RA are comparable. These data suggest that IL- 6 is the central factor for the disturbed homeostasis between bone resorption and bone formation in the joints of RA patients.

Acknowledgements: The RE-BONE study was supported by Chugai Pharmaceutical Co., Ltd.

Disclosure of Interest: None declared

DOI: 10.1136/annrheumdis-2018-eular.3188

\section{FRI0051 THE RISK OF ASEPTIC ARTHROPLASTY LOOSENING IN PATIENTS WITH RHEUMATOID ARTHRITIS}

C. Böhler ${ }^{1}$, P. Weimann ${ }^{1}$, F. Alasti ${ }^{2}$, J.S. Smolen ${ }^{2}$, R. Windhager ${ }^{1}$, D. Aletaha ${ }^{2}$. ${ }^{1}$ Orthopaedics and Traumatology, ${ }^{2}$ Rheumatology, Medical University Of Vienna, Vienna, Austria

Background: Total joint arthroplasty (TJA) of the hip (THA) and knee (TKA) are well-established operations for end stage degenerative or inflammatory joint disease, and has excellent outcomes. In rheumatoid arthritis (RA), it is performed in about $25 \%$ of the patients. ${ }^{1}$ According to registry data septic complications after TJA are more frequent in RA than in osteoarthritis (OA), which is likely linked to the immunomodulatory therapy that RA patients receive. ${ }^{2}$ However, aseptic pros thesis loosening (APL) is the most common complication and it remains unclea whether RA per se is also a risk factor for non-infectious complications, e.g. by the presence of higher levels of systemic inflammation.

Objectives: To compare the rates of APL between OA and RA patients, and to investigate the influence of disease activity levels on the risk for APL in RA patients.

Methods: We identified all patients who underwent THA and TKA between 2002 and 2015 at our academic centre, and linked them with an existing prospective RA database to identify documented RA patients. Age and sex-matched OA patients were used as controls. Our primary endpoint were radiographic signs of APL as previously established. ${ }^{3,4}$ Radiographs were evaluated by two independent observers blinded to the clinical diagnosis.

To explore the effects of systemic inflammation, we compared the time integrated level of disease activity by the Simplified Disease Activity Index (SDAI) during the year before an $\mathrm{x}$-ray indicated loosening (for those with loosening) with the respective levels over one year before the last available $\mathrm{x}$-ray (for those without loosening).

We used nonparametric tests and the chi-square test to compare rates of loosening between RA and OA patients and to compare AUC SDAI between patients with and without APL. Additionally, we calculated a Cox proportional hazard 